\title{
Reporte del vampiro de patas peludas Diphylla ecaudata Spix, 1823 (Chiroptera: Desmodontinae) para el Chocó biogeográfico colombiano
}

\author{
Reports of the hairy-legged vampire bat Diphylla ecaudata Spix, 1823 \\ (Chiroptera: Desmodontinae) for the biogeographyc Colombian Chocó
}

\author{
Echavarría-R Jonard David ${ }^{1 *}$ Biol; Pino-M Yessica Luz¹ Biol; Rengifo-M Jhon Tailor ${ }^{1}$ Ph.D; \\ Sánchez-L Nancy² Biol; Quinto-M Jilbher² Biol. \\ ${ }^{1}$ Universidad Tecnológica del Chocó, Facultad de Ciencias Básicas y Exactas, \\ Programa de Biología, Grupo de Investigación en Herpetología, Colombia. \\ ${ }^{2}$ Universidad Tecnológica del Choco, Facultad de Ciencias Básicas y Exactas, Programa de Biología, \\ Grupo de Investigación Manejo de Fauna Silvestre Chocoana, Colombia.
}

\section{Keywords:}

Tropical dry forest; Chocó Department; geographical distribution; vampire bats; vampire.

\section{Palabras Clave:}

Bosque seco tropical; departamento del Chocó; distribución geográfica; murciélagos hematófagos; vampiro.

\section{Abstract}

It is reported a new record of the hairy-legged vampire Diphylla ecaudata (SPIX, 1823 ) in the Choco region of Colombia, of which are known only few studies in the country, registered in the departments of Magdalena (1900), Vaupés (1966), Meta (1970), Caquetá (1999) y Amazonas (2016). This registry is based on two specimens that were properly analyzed and due to its curiosity, discusses the available information on this species in the country in terms of its distribution and ecology, based on specimens in collections and existing reports. This log helps fill a void of the known geographic distribution of the species in the country deposited in the Teriologica Scientific Collection Research Group on Management and Wildlife Management of the Chocó of the Technological University of Chocó (ColTer-Choco). This report extends the geographic distribution until the department of Choco, in contraendose to $464.794 \mathrm{~km}$ some of the record closest in Magdalena, adding the Tropical Dry Forest Ecosystem (BST) to habitats that occurred for the species. Due to its curiosity, discusses the available information on this species in the country in terms of its distribution and ecology, based on specimens in collections and existing reports. This log helps fill a void of the known geographic distribution of the species in the country.

\section{Resumen}

Se reporta un nuevo registro del vampiro de patas peludas Diphylla ecaudata (SPIX, 1823) en el Chocó Biogeográfico colombiano, del que solo se conocen pocos estudios en el país, registrada en los departamentos de Magdalena (1900), Vaupés (1966), Meta (1970), Caquetá (1999), Casanare (2013) y Amazonas (2016). Este registro se basa en dos especímenes que fueron debidamente analizados y depositados en la Colección Científica Teriológica del Grupo de Investigación en Manejo y Gestion de la Vida Silvestre del Chocó de la Universidad Tecnológica del Chocó (ColTer-Chocó). Este reporte amplía la distribución geográfica hasta el departamento del Chocó, en contraendose a $464.794 \mathrm{~km}$ aproximadamente del registro más cercano en el Magdalena, adicionando el ecosistema Bosque Seco Tropical (BST) a los hábitats ocurridos por la especie. Debido a su curiosidad, se discute la información disponible sobre esta especie en el país en términos de su distribución y ecología, a partir de ejemplares en colecciones y reportes existentes. Este registro ayuda a llenar un vacío de la distribución geográfica conocida de la especie en el país. 


\section{Introducción}

Diphylla ecaudata (SPIX, 1823) (Fig. 1), es un murciélago de la familia Phyllostomidae de menor tamaño en relación con las otras especies de vampiros existentes como Desmodus rotundus (GEOFFROY, 1810) y Diaemus youngii (JENTINK, 1893), ampliamente distribuidas en la Región Neotropical (VILLA-R. 1976; GREENHALL et al. 1983; KOOPMAN, 1988; KWON y GARDNER, 2008; UIEDA, 2008). La especie en estudio presenta un rostro muy corto, con la hoja nasal reducida a una simple proyección redondeada, orejas cortas y anchas, ojos grandes y brillantes (GRENHALL et al. 1984, TERÁN y AGUIRRE, 2007). Además de presentar un color café oscuro y entre los detalles que la diferencian de las otras especies de vampiros, es que presentan las orejas ligeramente más pequeñas y redondeadas, el pulgar es corto, grueso en la base y sin callosidades. Sus miembros posteriores son más cortos que en los otros vampiros; sin embargo, las garras son más desarrolladas. Presenta un pelaje denso que cubre casi todo el animal, siendo la zona del uropatagio extremadamente densa (GREENHALL y SCHMIDT, 1996). En la cara, los pelos son más escasos y están limitados a los espacios entre las orejas, los ojos y la hoja nasal.

Esta especie es considerada la segunda en importancia, después de Desmodus rotundus, tanto en número de individuos como en su distribución (VIZOTTO y TADDEI, 1973). Esta rara especie presenta una amplia distribución geográfica a partir del sur de Texas, pasando por México oriental, parte de América Central hasta América del Sur, parte de Perú (QUITANA y PACHECO, 2007), Bolivia
(SILES et al. 2003), y Brasil, con abundancia en la región amazónica (VILLA, 1968, PERACCHI et al. 2006). En Venezuela se distribuye por Mérida (OJASTI y LINARES, 1971) y en Sucre en Cumaná (HANDLEY, 1976). En Ecuador en Pastaza, Mera (ALBUJA, 1983).

En Colombia, Diphylla ecaudata es conocida por muy pocos registros, encontrándose en los departamentos de Magdalena (ALLEN, 1900), Vaupés (WENZEL et al. 1966), Meta (AELLEN, 1970), Caquetá (MONTENEGRO y ROMERO-RUIZ, 1999), Casanare (ABC, 2013) y Amazonas (CASTRO-CASTRO, 2016) (Fig. 2). Esta especie habita desde bosques tropicales húmedos, en altitudes que van desde cero hasta 1020 metros sobre el nivel del mar, con presencia de la especie en formaciones de Bosque Seco Tropical (BST), como lo reportamos aquí, con temperaturas entre los 23 y $28^{\circ} \mathrm{C}$ (GREENHALL et al. 1984). Este reporte amplía la distribución geográfica hasta el departamento del Chocó, en contraendose a $464.794 \mathrm{~km}$ aproximadamente del registro más cercano en el Magdalena.

Este ejemplar de $D$. ecaudata fue colectado en el marco del Proyecto de la CTel para el mejoramiento del Sector Maderero en el departamento del Chocó", donde se realizó la caracterización y monitoreo de los Quirópteros, como potenciales dispersores de semillas de plantas maderables en el Chocó. En este estudio se empleó el método de redes de nieblas, siguiendo los métodos de base en BORELL (1937), NELSON (1965), WHITAKER (1972) y KUNZ et al. (2009) con algunas modificaciones propuestas por LOAYZA et al. (2006), KUNZ y WEISE (2009), con pequeños ajustes

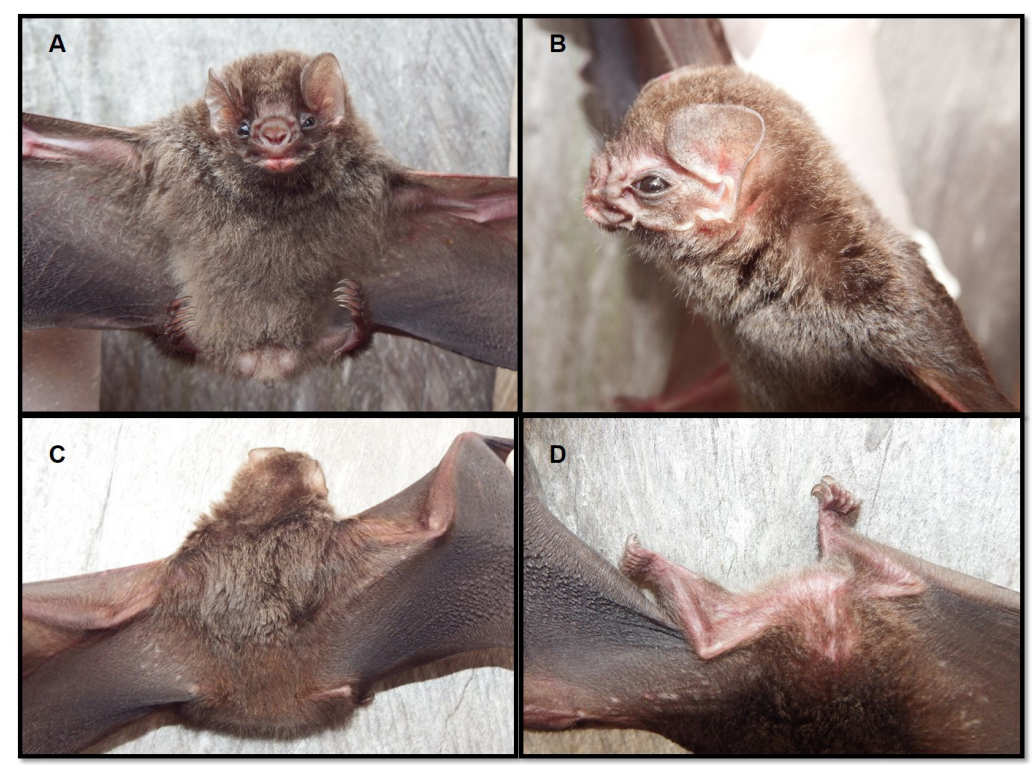

Figura 1. Espécimen de Diphylla ecaudata colectado en la localidad de Acandí Seco Alto, municipio del Acandí, Chocó - Colombia. Ventral (A), lateral (B), dorsal (C) e llustrando de las patas peludas. Foto: Jonard David Echavarría-Rentería. 
de acuerdo a las condiciones ambientales y del terreno en el área de estudio. Se realizaron dos expediciones a la localidad de Acandí Seco Alto, municipio de Acandí, donde se capturaron dos individuos de $D$. ecaudata. El primer ejemplar $\left(8^{\circ} 22^{\prime} 46.81^{\prime \prime} \mathrm{N}, 77^{\circ} 19^{\prime} 25.82^{\prime \prime} \mathrm{W}, 1020\right.$ msnm) corresponde a un macho adulto encontrado el 8 de diciembre de 2015, colectado por la Bióloga Nancy Sánchez Lozano, entre las 5:00 y 6:00 h de la madrugada y presencia de una fina llovizna, a una altura aproximada de 3 metros. En otra visita a la misma zona se observó nuevamente el segundo individuo colectado por el Biólogo Jonard David Echavarría-Rentería el 16 de marzo de 2016. Este ejemplar fue observado a las 23:12 h con presencia de llovizna, igualmente en una noche con mucha luz; según el calendario lunar, la luna se encontraba en cuarto creciente.

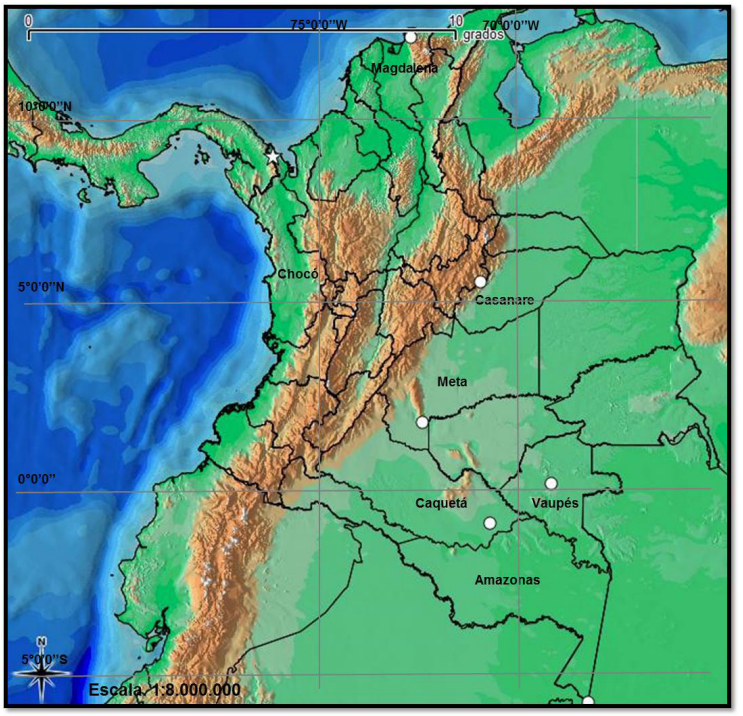

Figura 2. Distribución geográfica del vampiro de patas peludas Diphylla ecaudata en Colombia. Los Círculos Blancos corresponden a reportes de literatura. La Estrella Blanca correspondes al Nuevo registro para el Chocó biogeográfico colombiano presentado aquí. Fuente: Mapa obtenido de QGIS (Sistema de Información

Estos individuos fueron preservados y depositados en la Colección Teriológica y Ornitológica del Grupo de Investigación en Manejo de Fauna Silvestre Chocoana de la Universidad Tecnológica del Chocó (ColTerOrni), con número de colección (CMCH001759) y (CMCH001760). Los ejemplares aquí capturados coinciden con las características y medias registradas para la especie por GREENHALL et al. (1984); KOOPMAN (1988); KWON y GARDNER (2008) (Tabla 1).

Adicionalmente, se revisó la base de datos de la colección biológica en línea del Instituto de Ciencias Naturales de la Universidad Nacional de Colombia (ICN), de donde se obtuvo registros de presencia de la especie en territorio colombiano, y junto a nuestro registro fue utilizado para construir un mapa de puntos de presencia demostrada para la especie.

Tabla 1. Medidas externas y craneales de los ejemplares examinados.

\begin{tabular}{ccc}
\hline Descripción & (CMCH001759) & (CMCH001760) \\
\hline LCC & $60 \mathrm{~mm}$ & $61,28 \mathrm{~mm}$ \\
AB & $34,35 \mathrm{~mm}$ & $53,74 \mathrm{~mm}$ \\
3MC & $22,01 \mathrm{~mm}$ & $27,94 \mathrm{~mm}$ \\
LMC & $21,04 \mathrm{~mm}$ & $22,41 \mathrm{~mm}$ \\
Ltra & $5,03 \mathrm{~mm}$ & $7,54 \mathrm{~mm}$ \\
LP & $9,25 \mathrm{~mm}$ & $11,89 \mathrm{~mm}$ \\
Falange & $6,15 \mathrm{~mm}$ & $11,02 \mathrm{~mm}$ \\
LLT & $127,25 \mathrm{~mm}$ & $134,81 \mathrm{~mm}$ \\
Peso & $27 \mathrm{gr}$. & $39 \mathrm{gr}$. \\
\hline
\end{tabular}

Longitud Total del Cuerpo (LCC), Medida de Antebrazo, Tercer Metacarpiano, Longitud Mayor Cráneo, Longitud el Trago, Longitud del Pie, Longitud Total del Animal.

MONTENEGRO y ROMERO-RUIZ (1999), SILES et al. (2003) y ACOSTA (2006), hallaron que la mayoría de los registros de $D$. ecaudata se encuentran en áreas protegidas, por lo general en bosques bien conservados, cuevas o en Parques Nacionales Naturales. Pero en esta investigación observamos que esta especie se colectó en un bosque intervenido por actividades antropogénicas, como la minería y la tala de árboles, además por el aprovechamiento forestal, la agricultura y la ganadería. Esta última permite inferir, que esta especie aparte de alimentarse de la sangre fresca de las aves (GREENHALL y SCHUTT, 1996), también puede consumir sangre de mamíferos. RUSCHI en 1951 observó que esta especie en cautiverio puede casualmente consumir sangre de mamíferos tales como porcinos, bovinos y equinos.

$D$. ecaudata es una especie rara que no se observa con frecuencia, conociéndose solo por pocos registros mostrados en el país, encontrados en zonas rurales y selváticas, como la zona amazónica (Caquetá, Vaupés y Amazonas), en los llanos orientales (Meta y Casanare) y la región de la costa atlántica (Magdalena) y en el departamento del Chocó aportado en este estudio. Esta especie a pesar de los escasos registros en el país actualmente se encuentra clasificada en la categoría preocupación menor (LC por su sigla en inglés) de la Unión Internacional para la Conservación de la Naturaleza (IUCN, 2016 por sus siglas en inglés).

Este estudio aporta información ecológica de los hábitats ocurridos por la especie ya que explora cavernas como principal abrigo, siendo raramente encontrados en huecos de árboles, hornos de carbón, minas y casas abandonadas. Esto demuestra fuerte selección de abrigos, siendo una posible explicación para su relativa escasez en la naturaleza (UIEDA, 1996). Además de ampliar la distribución geográfica en la región atlántica, norte del departamento del Chocó, hechos que facilitarían la elaboración de planes de conservación 
con soporte científico y mapas de distribución para el país, permitiendo identificar áreas convenientes para la conservación de esta especie. Debe destacarse que mayores estudios son aún necesarios para establecer la distribución de la especie en el departamento, una región que a pesar de los impactos antrópicos mantiene algunas áreas con una importante biodiversidad.

Agradecimientos: Esta investigación fue financiada con recursos de regalías (Gobernación del Chocó) y ejecutado por la Universidad Tecnológica del Chocó "Diego Luis Córdoba" en el marco del proyecto "Aplicación de la Ciencias y Tecnologías para el
Mejoramiento del Sector Maderero en el departamento del Chocó", bajo la dirección general del ingeniero Yesid Emilio Aguilar Lemus. Agradecemos a la Universidad Tecnológica del Chocó, por ser una vez más una institución que apoya las investigaciones científicas en el departamento del Chocó, además por todo lo enseñado durante los estudio de pregrado, a Leison Palacios-Mosquera del Grupo de Investigación en Manejo de Fauna Silvestre Chocoana (FASCHO) por la identificación de la especie y al grupo de Investigación en Recursos Vegetales y por último a la comunidad de Acandí Seco del municipio de Acandí por su hospitalidad.

\section{Referencias}

ACOSTA, L. 2006. Inventariación, Historia Natural y Aportes Biogeográficos de la Quirópterofauna del Bosque Experimenta Elías Meneses, Santa Cruz-Bolivia. Tesis de licenciatura. Universidad Autónoma Gabriel Rene Moreno. Santa Cruz, Bolivia.

AELLEN, V. 1970. Catalogue raisonné des Chiroptères de la Colombie. Revue Suisse de Zoologie 77:1-37.

ALBUJA, V.L. 1999. Murciélagos del Ecuador. 2nd ed. Cicetrónica Cía. Ltda. Offset. Quito, Ecuador.

ALLEN, J.A. 1900. List of bats collected by Mr. H. H. Smith in the Santa Marta region of Colombia, with descriptions of new species. Bulletin of the American Museum of Natural History 13:87-94.

ASOCIACIÓN DE BECARIOS DEL CASANARE - ABC. 2013. Inventarios de Murciélagos de la Microcuenca de la Quebrada Agua Blanca, Vereda Marroquín, Yopal, Casanare.

BOREL, E. 1937. Valeur Pratique et Philosophie des Probabilités, Paris: Gauthier-Villars.

CASTRO-CASTRO, F.F. 2016. Nuevo reporte del murciélago hematófago de patas peludas Diphylla ecaudata SPIX, 1823 (Chiroptera, Phyllostomidae) en Colombia. Mastozoología Neotropical 23(2):529-532.

GEOFFROY, É. 1810. Sur les Phyllostomes et les Mégadermes. Ann. Mus. Hist., Nat. 15:157-198.

GREENHALL, A.M.; JOERMANN G.; SCHMIDT, U. 1983. Desmodus rotundus. Mammalian Species 202:1-6.

GREENHALL, A.M.; SCHMIDT U.; JOERMANN, G. 1984. Diphylla ecaudata. Mammalian Species 227:1-3.

GREENHALL, A.M.; SCHMIDT, U.; JOERMANN, G. 1984. Diphylla ecaudata. Mammalian Species 227: 1-3.

GREENHALL, A.M.; SCHUTT, W.A. 1996. Diaemus youngi. Mammalian Species 533: 1-7.

HANDLEY, C.O., Jr. 1976. Mammals of the Smithsonian Venezuelan Project. Brigham Young University Science Bulletin, Biological series 20 (5):1-89.

IUCN (International Union for Conservation of Nature) 2016. Diphylla ecaudata. The IUCN Red List of Threatened Species. Versión 2016-2.

JENTINK, L.A. 1893. On a collection of bats from the West Indies. Notes of the Leyden Museum, 15:278-283. 
KOOPMAN, K.F. 1988. Systematics and distribution. Págs. 7-17, en: Greenhall, A.M.; Schmidt, A.M (Eds.). The natural history of vampire bats. CRC Press. Boca Raton, EEUU.

KUNZ, T.H.; WEISE, C.D. 2009. Methods and Devices for Marking Bats. Págs. 36-56. En: Kunz, T.H.; Parsons, S. (Eds.). Ecological and Behavioral Methods for the Study of Bats. Second edition. The Johns Hopkins University Press. Baltimore, EE.UU.

KWON, M., GARDNER, A.L. 2008. Subfamily Desmodontinae. Págs. 239-245. En: Gardner, A.L. (Ed.). Mammals of South America, vol. 1, Marsupials, Xenarthrans, Shrews, and Bats. The University of Chicago Press. Chicago, EEUU.

MONTENEGRO, O.L.; ROMERO-RUIZ, M. 1999. Murciélagos del sector sur de la serranía de Chiribiquete, Caquetá, Colombia. Revista Academia Colombiana Ciencia 23:641-649.

NELSON, J.E. 1965. Behaviour of Australian Pteropididae (Megachiroptera.) Animal Behaviour 13:544-557.

OJASTI, J.; LINARES, O.J. 1971. Adiciones a la fauna de murciélagos de Venezuela con notas sobre las especies del género Diclidurus (Chiroptera). Acta Biológica Venezuelica 7:421-41.

QUINTANA, H.; PACHECO, V. 2007. Identificación y distribución de los murciélagos vampiros del Perú. Revista Peruana de Medicina Experimental de Salud Pública 24 (1):81-88.

RUSCHI, A. 1951. Morcegos do Estado do Espírito Santo: Descrição de Diphylla ecaudata Spix e algumas observações a seu respeito. Boletim do Museu de Biologia Prof. Mello Leitão 3:1-6.

SILES, L.; MUÑOZ, A.; AGUIRRE, L.F. 2003. Nuevos reportes del vampiro de patas peludas Diphylla ecaudata (Chiroptera: Desmodontinae) para los Departamentos de Cochabamba y Santa Cruz. Ecología en Bolivia 38:141-145.

SPIX, JDE. 1823. Simiarum et Vespertilionum brasiliensium species novae, p. 68. Monachii, 72 pp.

TERÁN, M.; AGUIRRE, L.F. 2007. Subfamilia Desmodontinae. Págs. 293-297. En: Aguirre, L.F. (Ed.). Historia Natural, Distribución y Conservación de los Murciélagos de Bolivia. Fundación Simón I. Patiño, Santa Cruz, Bolívia.

UIEDA, W. 1996. Biologia e dinâmica populacional de morcegos hematófagos, Págs. 63-87. Anais do II Curso de Atualização em raiva dos herbívoros. Curitiba, Brasil.

UIEDA, W. 2008. História natural dos morcegos hematófagos no Brasil. Págs. 179-198, En: Pacheco, S.M.; Marques, R.V.; Esberard, C.E.L. (Eds.). Morcegos no Brasil: biologia, sistemática, ecologia e conservação. Armazem Digital. Porto Alegre, Brasil.

VILLA, B. 1968. Ethology and ecology of vampire bats. International Union for Conservation of Nature Publications. n. serie Morges 13:104-110.

VILLA-RAMíREZ, B. 1976. Biología de los murciélagos hematófagos. Págs. 94-99, en: México: Laboratorio de Mastozoología, Instituto de Biología UNAM. Recuperado de http://www.fmvz.unam.mx/fmvz/ cienciavet/revistas/ CVvol1/CV1v1c04.pdf.

VIZOTTO, L.D.; TADDEI, V.A. 1973. Chave para a determinação de quirópteros brasileiros. São José do Rio Preto, Brasil.

WENZEL, R.L.; LIPTON, V.J.; KIEWLICZ, A. 1966. The streblid batflies of Panama (Diptera, Calypterae: Streblidae). Págs. 405-676. En: Wenzel, R.L.; Lipton, V.J. (Eds.). Ectoparasites of Panama. Field Museum of Natural History. Chicago, EEUU.

WHITTAKER, R.H. 1972. Evolution and measurement of species diversity. Taxon, 21(2/3):213-251. 